\title{
Epigenome: The symphony in your cells
}

A slew of papers reveals the chemical tweaks to DNA in a wealth of different cells - as explained with the help of a small orchestra.

Kerri Smith

18 February 2015

Almost every cell in the human body has the same DNA sequence. So why is a heart cell different from a brain cell? Cells use their DNA code in different ways, depending on their jobs - just as the orchestra in this video can perform one piece of music in many different ways. The combination of changes in gene expression in a cell is called its epigenome.

For more than a decade, scientists have had access to a reference human genome. Now, the equivalent for the epigenome has been published, in a collection of papers appearing on 18 February in Nature and several other journals. A large international group of researchers has put together 111 epigenomes from different human cell types, including all the major organs, immune cells and embryonic stem cells. The team has also assembled epigenomes for induced pluripotent stem cells — cells that are derived from adult cells and coaxed into becoming capable of developing into almost any other type of cell in the body.

The researchers looked for features including chemical tweaks to DNA that prime genes to be switched on or off, and alterations to the 'histone' proteins around which DNA is wrapped. Chemical or structural modifications to histones can affect which genes the cellular machinery translates into proteins and which remain silent. Such epigenetic changes can dramatically affect a cell's behaviour and function.

The epigenomes also contain hints of how epigenetic changes could be involved in diseases, including cancer, Alzheimer's disease and autoimmune diseases.

Taken together, the work demonstrates how a cell's epigenome is complex and exquisitely arranged — just like a Beethoven symphony. 